\title{
Maestría en la Docencia de las Matemáticas
}

\section{La variación en libros de texto de álgebra escolar}

Autor: OTÁLORA L; Rosa Inés

Palabras claves: Variación, Variable, Letra, Pensamiento Variacional, Texto.

\section{DESCRIPCIÓN}

El estudio presenta un análisis del tratamiento didáctico de las nociones de variación y variable en libros de texto de álgebra escolar, para lo cual se revisan y comparan dos series de textos de grado sexto a octavo.

\section{CONTENIDO}

En el Capítulo 1 se presentan los antecedentes de la investigación, el problema, la justificación y los objetivos tanto generales como específicos. En el Capítulo 2 se desarrolla el marco teórico que soporta la investigación, se inicia con la clasificación de las letras en el algebra para luego profundizar en el uso de la letra como variable; en seguida se desarrollan las nociones de variación y pensamiento variacional y, finalmente se hace mención de cómo es presentado el pensamiento variacional en los Lineamientos Curriculares. El Capítulo 3 está dedicado a la metodología, la cual se basa en la aplicación de tres tipos de análisis para la caracterización de los libros de texto: análisis conceptual, análisis didáctico cognitivo y análisis fenomenológico. En el Capítulo 4 se presentan el análisis y resultados del estudio realizado a las dos series de textos, con base en las tres categorías de análisis definidas en el capitulo 3, y en el Capítulo 5 las conclusiones.

\section{METODOLOGÍA}

Para este estudio se estudiaron dos series de texto de matemáticas para grados sexto a octavo. En cada una de ellas se realizaron tres tipos de análisis, a saber: El análisis conceptual que busca determinar cómo se define y organizan los concepto a lo largo del texto; el análisis didáctico cognitivo que se refiere a analizar el modelo de enseñanza predominante en el texto, la concordancia del texto con los Lineamientos Curriculares y si existe coherencia entre los estándares que el texto propone desarrollar y el contendido de las unidades temáticas correspondientes; y el análisis fenomenológico que busca determinar los fenómenos a los que recurren los ejemplos y situaciones problemas del texto con respecto al concepto de variable y el desarrollo del pensamiento variacional.

\section{CONCLUSIONES}

La introducción desde los primeros años escolares de situaciones que propicien el reconocimiento del cambio y la generalización de patrones, permitirá que los estudiantes se familiaricen con este tipo de situaciones y que por ende les resulte más fácil comprender la noción de variación cuando sea el momento de formalizarla y utilizarla en la modelación de situaciones problema. 
Algunas de las situaciones presentadas en las dos series de textos analizadas se pueden utilizar o transformar para introducir la noción de variación y para fomentar el desarrollo del pensamiento variacional. Sin embargo, los textos no presentan explícitamente la forma como esto se puede lograr ni hay otro documento que lo haga. Corresponde entonces al docente tener una visión crítica y proactiva que le permita, de una parte aprovechar las fortalezas del texto que tiene en sus manos y de otra reconocer las debilidades del mismo y transformarlas en oportunidades de enseñanza para sus estudiantes.

Una herramienta poderosa para iniciar a los estudiantes en el reconocimiento de situa- ciones de cambio es el uso de los diferentes sistemas de representación asociados a la variación, como lo son las representaciones tabulares, las representaciones pictóricas o icónicas, las gráficas y los enunciados verbales, entre otros.

Dentro del desarrollo de los contenidos de cada unidad no siempre se presentan de manera explícita todos los elementos necesarios para alcanzar los estándares propuestos al inicio de las mismas. Tampoco se advierte al docente de esta situación ni se le orienta en la forma como puede lograr, con los elementos presentes en la unidad, alcanzar los estándares y competencias que se tienen previstos.

\section{Características de talento matemático asociadas a la visualización en contextos algebraicos.}

Autor (s): JIMÉNEZ GÓMEZ, William Alfredo; ROJAS TOLOSA, Sandra Milena

Palabras clave: Visualización, Talento.

\section{DESCRIPCIÓN}

Este trabajo va dirigido a aquellos docentes de matemáticas interesados en el tema del talento matemático, en especial en los aspectos relacionados con su definición e identificación. Se encuentran aspectos generales relacionados con la caracterización del talento matemático y el proceso de visualizar, este último analizado desde el álgebra; además se presentan ejemplos que dan cuenta de las relaciones existentes entre los elementos propios de la visualización y las características asociadas al talento matemático. Lo cual se determinó con base en la interpretación de soluciones a pro- blemas presentadas por estudiantes entre 13 y 16 años nominados como talentosos en matemáticas que participaban en un proyecto de intervención en la Universidad Pedagógica Nacional.

\section{CONTENIDO}

El trabajo se compone de tres capítulos:

El primero comprende los antecedentes de investigación relacionados con visualización y talento matemático y, el planteamiento del problema. 
En el segundo capítulo se presenta el marco teórico en dos apartados principales. En el primero, los diferentes modelos de talento que se encuentran relacionados con el talento matemático y de los cuales es posible inferir una definición basada en una caracterización, siendo la visualización un aspecto relevante en la identificación del talento matemático. De allí se inicia el segundo apartado que comprende los referentes teóricos de la visualización: definición y elementos (imágenes, habilidades y procesos); la interpretación de los diferentes elementos se hace desde el álgebra puesto que en los documentos consultados se encuentra enfocada, en su mayoría, en el campo de la geometría.

En los dos primeros apartados del tercer capítulo, se describe el enfoque de investigación en el cual se enmarca el estudio y una descripción detallada de las acciones que se tomaron para, a partir del instrumento utilizado, hacer el análisis de resultados presentados en el tercer apartado. Los resultados se analizaron empleando análisis estadístico a través de la elaboración y análisis de gráficos que muestran la frecuencia de aparición de imágenes, habilidades y procesos propios de la visualización, al igual que la frecuencia de aparición de características de talento matemático evidenciadas en los mismos segmentos de transcripción; a partir de esta información se elaboraron otro tipo de gráficos que representan la correlación entre los aspectos mencionados anteriormente, en cumplimiento del objetivo general del trabajo. Adicionalmente, se exponen como ejemplo transcripciones de clase donde se evidencian características de talento matemático y los elementos de la visualización en la presentación de los estudiantes de la resolución de un problema. Finalmente se presentan las conclusiones y algunas recomendaciones tanto desde el punto de vista pedagógico como investigativo.

\section{METODOLOGÍA}

El trabajo se desarrolla bajo el enfoque interpretativo y se enmarca dentro de la investigación "El Club de Matemáticas de la Universidad Pedagógica Nacional: Un espacio para identificar talentos matemáticos", financiada por el Centro de Investigaciones de la Universidad Pedagógica Nacional (CIUP) desarrollada en durante el 2008 y 2009. En el marco de esta investigación se diseñó un curso para el primer semestre del 2009.

Durante el desarrollo del curso se utilizaron diferentes instrumentos para la recolección de información sobre el desempeño de los estudiantes del curso, uno de ellos fue el registro en video de cada una de las actividades desarrolladas en el aula. Este instrumento se constituyó en la base para la realización de este trabajo; así, se realizó un análisis de los videos de clase para lo cual (i) se seleccionaron los videos a ser observados, (ii) se realizó una descripción general de los momentos en donde se evidenciaba la característica visualización en la presentación de la solución de los problemas planteados a los estudiantes, (iii) se hizo la descripción particular de los videos $y$, por último, (iv) se eligieron las transcripciones de los videos en cuestión.

Finalmente se recurre al análisis de las transcripciones seleccionadas para establecer cuáles características de talento matemático están relacionadas con los elementos de la visualización y ejemplificar cada una de las relaciones.

\section{CONCLUSIONES}

Las conclusiones generales obtenidas del trabajo son las siguientes:

Se encontró en la revisión documental que la manera más común de referirse al talento matemático es a partir de la caracterización; 
sobre este aspecto se establecieron relaciones entre las diferentes caracterizaciones que han propuesto diversos autores, concluyendo que todas las caracterizaciones posteriores a la elaborada por Wederlin (1958 citado por Krutetskii, 1976) son particularizaciones de ésta lo cual se considera un aporte a la didáctica matemáticas, en relación al talento matemático, pues esta organización permite conjeturar la existencia de relaciones o jerarquías entre las características de talento matemático, en consecuencia, los programas de intervención podrían definirse con base a las características más generales. El presente trabajo aporta en esta dirección, puesto que muestra que la visualización puede ser considerada como una característica de nivel superior, ya que se establecen relaciones entre algunos de sus elementos: discriminación visual, identificación visual y procesamiento visual y las características generalización, organización de información y flexibilidad. Estas relaciones son:

\section{Generalización y Discriminación Visual.} Cuando los estudiantes hacen discriminación visual hacen una comparación de varios objetos identificando sus semejanzas y diferencias visuales lo que está relacionado con la generalización, pues según Mason, Graham, Pimm y Gowar (1993 citados en García, Sánchez y Mora, 2009), este proceso contiene cuatro fases: ver, describir, escribir y verificar; la primera se caracteriza por "ver la regularidad, las relaciones y diferencias y se constituye en el proceso mental por el cual se distingue lo que es propio de cada situación y lo que es común a todo, lo que no varía. Para esta etapa es necesario reparar en las figuras que se tiene e intentar mostrar la que sigue la secuencia, considerando así características que puedan no ser perceptibles a simple vista" (García et al., 2009 , p. 3). De aquí que un indicador de la generalización es la discriminación visual pues sin ésta no es posible dar paso a las fases siguientes.

Organizar la información y Procesamiento visual. Cuando un estudiante procesa visualmente, transforma información abstracta a una imagen o una imagen a otra imagen, lo que le permite solucionar problemas de manera efectiva; éste es uno de los descriptores de organización de información que se describe como la capacidad para organizar la información, relacionarla y hacer uso de los datos e información de manera eficiente. Esta relación y las evidencias encontradas permiten afirmar que cuando existe procesamiento visual tuvo que organizarse la información dada y establecer relaciones.

Flexibilidad e Identificación Visual 2. Uno de los indicadores que permite afirmar que una persona tiene la característica flexibilidad, es la desarticulación de esquemas rígidos, que consiste en descomponer el todo en sus partes. En este sentido, hay una relación directa con la identificación visual 2 descrita como: reconocer un objeto aislándolo de su contexto, dado que para aislar un objeto de su contexto se hace necesario desarticular un esquema. Sin embargo, es importante aclarar que aunque exista esta relación entre la flexibilidad y la identificación visual 2, la aparición de esta última no implica la flexibilidad, pues la desarticulación de un esquema es sólo una parte del proceso, puesto que después de haber hecho una desarticulación se hace necesario establecer relaciones innovadoras entre las partes para hacer evidente la flexibilidad.

De la revisión documental se concluye que, en cuanto a las investigaciones relacionadas tanto con el tema de la visualización como la caracterización de talento matemático, se evidencia que éstas se enmarcan en la clasificación de estudiantes talentosos en matemáticas en tres tipos: visualizadores, 
no visualizadores y armónicos, sin hacer explícitas las características de cada uno de estos tipos; además, se hace un reconocimiento a la importancia de la resolución de problemas como un instrumento idóneo para la caracterización de estudiantes con talento matemático; en este mismo sentido, la mayoría de autores (Wederlin 1958; Krutestkii, 1976; Greenes,1891; Freiman, 2006, Benavides, 2008) coinciden en que la capacidad para solucionar problemas matemáticos en los que se involucran diversas habilidades está directamente relacionada con el talento matemático.

Por otro lado, el análisis de la presentaciones que hacen los estudiantes talentosos permite confirmar lo propuesto por Kru- testkii (1976) en cuanto a las habilidades que caracterizan la destreza matemática; la identificación de los aspectos que enmarcan cada una de estas habilidades solamente es posible a través de la observación de conductas, desempeños, habilidades o estrategias utilizadas cuando los estudiantes resuelven problemas matemáticos.

En consecuencia, las relaciones establecidas entre algunos elementos de la visualización y algunas características de talento permiten afirmar que los problemas en donde se hace necesaria la visualización para dar una solución, son una herramienta efectiva para identificar características de talento matemático como la generalización, la organización de información y la flexibilidad

\section{Análisis de las competencias matemáticas asociadas al concepto de número racional en libros de texto de grado séptimo.}

Autores: GIRALDO, O. y MARTÍNEZ, C.

Palabras claves: Competencias matemáticas, concepto de número racional, competencia numérica, libros de texto.

\section{DESCRIPCIÓN}

El estudio presenta un análisis de la competencia numérica asociada al concepto de número racional en los textos escolares, para ello se analizan dos textos de grado séptimo.

\section{CONTENIDO}

En el Capítulo 1 se presentan la revisión de la literatura, la justificación, el problema de investigación y los objetivos tanto generales como específicos. En el Capítulo 2 se desarrolla el marco de referencia para el análisis, se describen y analizan los aspectos matemáticos del número racional y los significados asociados; enseguida se describen y analizan los aspectos metodológicos de los libros de texto, se profundiza en las competencias matemáticas, en particular se realiza una revisión a la competencia numérica en los Principios y Estándares para la Educación Matemática, en los Estándares Básicos de Competencias y en las pruebas SABER y PISA, y se analiza la noción de contexto; por último se analiza el lugar que ocupa el número racional 
en los Lineamientos Curriculares de Matemáticas. El Capítulo 3 está dedicado a la metodología y al análisis, se presenta la unidad de análisis del estudio: invariantes del concepto de número racional (relaciones de equivalencia y orden), las competencias numéricas asociadas y el análisis metodológico. Por último, se presenta el análisis de los textos. En el Capítulo 4 se encuentran las conclusiones divididas de acuerdo con las categorías que se tuvieron en cuenta para el análisis.

\section{METODOLOGÍA}

El enfoque metodológico de investigación es del tipo descriptivo interpretativo, asumiendo como herramienta el análisis de contenido, acorde con la unidad de análisis, el cual se estructura mediante los siguientes componentes:

- El objeto de análisis: Para nuestro estudio, corresponde a dos libros de texto de grado $7^{a}$.

- Las unidades de análisis: Las unidades sobre los números racionales en los libros de texto escogidos para el estudio.

- Las categorías de análisis: Se establecen tres categorías de estudio para abordar el estudio: los invariantes asociados al concepto del número racional; análisis metodológico y las competencias asociadas al concepto de número racional que se promueven en la unidad analizada.

\section{CONCLUSIONES}

Algunas de las conclusiones que se destacan son las siguientes: Invariantes del concepto de número racional:

Los números racionales surgen por la necesidad de ampliar el dominio numérico tal y como se hace con el método genético, se hace uso de los significados (operador y cociente) para la construcción "didáctica" del concepto de número racional, sin embargo dicho concepto se ilustra mediante definiciones.

Se hace uso de la representación y comparación en la recta numérica para establecer las relaciones de equivalencia y orden.

Las competencias asociadas y que se promueven ponen el énfasis en las capacidades y en el uso de esas capacidades, las secciones de talleres y ejercicios exigen replicar lo desarrollado en los ejemplos.

Del análisis metodológico realizado en los textos se evidencia que la estructura general está compuesta por unidades, dicha estructura consta de una introducción, la organización de los contenidos tiene una estructura definida y repetitiva, de esta manera el aprendizaje que promueve es por repetición. Los ejercicios se apoderan de las actividades, siendo éstos los que se presentan en mayor medida, los problemas son relegados al final de la lección, son escasos y no contextualizados. 